\title{
INFLUENCES OF T-STRESS ON CONSTRAINT EFFECT IN MISMATCHED MODIFIED BOUNDARY LAYER MODEL FOR CREEP CRACK
}

\author{
Yanwei Dai \\ Department of Engineering Mechanics, AML, \\ Tsinghua University, \\ Beijing, China \\ e-mail:daiyw13@mails.tsinghua.edu.cn
}

Yinghua Liu*

Department of Engineering Mechanics, AML, Tsinghua University,

Beijing, China

Haofeng Chen

e-mail: yhliu@mail.tsinghua.edu.cn

Department of Mechanical \& Aerospace Engineering,

University of Strathclyde,

Glasgow, G1 1XJ, UK

e-mail: haofeng.chen@strath.ac.uk

\section{ABSTRACT}

Constraint effect plays an important role in assessing the stress field and the growth rate of creep crack in components under high temperature. The mismatched modified boundary layer (MMBL) model is extended to creep crack in this paper. For the MMBL model, the $Q$-parameters for different mismatch factors are studied under different $T$-stresses. The variation of the dimensionless $T$-stress in creep zone is given. The variations of open stresses with creep time for different mismatch factors are presented under different $T$-stresses. The comparisons of $Q$ parameter between homogeneous material and mismatched materials are made. The influences of mismatch factor on the constraint parameter are discussed. The influence of creep exponent on the open stress is also discussed.

\section{NOMENCLATURE}

$a_{i} \quad(i=1,2,3) \quad$ Coefficients of $Q-T$ relationship

$A_{\mathrm{B}} \quad$ Creep constant of power law creep for base metal

$A_{\mathrm{C}} \quad$ Unified constraint parameter given by Ma et al.

$A_{\mathrm{W}} \quad$ Creep constant of power law creep for base metal

$A_{2}^{*} \quad$ Constraint parameter given by Chao et al.

$\mathrm{C}(t) \quad C$-integral for transient creep

$C^{*} \quad C^{*}$-integral for extensive creep

\begin{tabular}{cl}
$E$ & Young's modulus \\
$J$ & J-integral \\
$K_{\mathrm{I}}$ & Stress intensity factor of mode I \\
& \\
$I_{n}$ & Integral constant for HRR field \\
& \\
$M_{\mathrm{P}}$ & Strength mismatch factor for plastic material \\
& \\
$M_{\mathrm{C}}$ & Creep mismatch factor \\
& \\
$n$ & Creep exponent \\
$Q$ & Constraint parameter \\
$r$ & Distance from crack tip \\
$T$ & $T$-stress \\
& \\
$u_{x}$ & Displacement in $x$-direction \\
$u_{y}$ & Displacement in $y$-direction \\
$v$ & Poisson's ratio \\
$\dot{\varepsilon}_{0}$ & Reference creep strain rate \\
$\sigma_{0}$ & Yielding stress \\
$\sigma_{i j}$ & Stress component \\
$\tilde{\sigma}_{i j}$ & Angular distribution function \\
$\sigma_{22}$ & Open stress \\
$\sigma_{22}^{\text {HRR }}$ & Open stress of HRR field \\
$\sigma_{22}^{\text {SYY }, T=0}$ & Open stress of small scale yielding \\
$\sigma_{22}^{\text {SSC,T=0}}$ & Open stress of small scale creep \\
$\theta$ & Polar angle \\
\hline &
\end{tabular}




\section{INTRODUCTION}

The mismatch effect of creep crack can affect the crack tip stress field greatly. The accurate prediction and better understanding of creep crack tip stress field is a basis to evaluate a mismatched creep crack. The so-called mismatch effect is mainly caused by the difference of materials. For an elastoplastic weldment, the mismatch effect is always characterized by a mismatch factor $M_{\mathrm{p}}$, which is defined by the ratio of yielding stress between weld metal and base metal, shown as below

$$
M_{\mathrm{p}}=\frac{\sigma_{\mathrm{yW}}}{\sigma_{\mathrm{yB}}}
$$

where $\sigma_{\mathrm{yW}}$ is the yielding stress of weld part and $\sigma_{\mathrm{yB}}$ is the yielding stress of base part. A lot of studies have been focused on the mismatch effect of elastoplastic material. The estimation of $J$-integral for a typical dissimilar material was presented by Haddi and Weichert [1]. The mismatch effect on the perfectly elastoplastic welding specimens was investigated by Kim and Schwalbe [2, 3]. Song et al. [4] presented the so-called mismatched limit load and $J$-integral approximation of surface flaw in a tensional plate. Except for the discussions of stress and fracture parameter estimation of crack tip for an elastoplastic material, there were also some researches on the constraint effect of mismatched weldments, e.g. Zhang et al. [5] gave a two parameter $J-M$ method to characterize the constraint effect for an interfacial crack. Similar investigations can be also seen in Refs. $[6,7]$.

The mismatch effect also exists in creeping weldments. However, the definition of mismatch factor for a creep welding component is rather different from an elastoplastic weldment. As usual, the definition of mismatch factor for creeping solids with an elastic power-law constitutive equation can be presented as

$$
M_{\mathrm{C}}=\left(\frac{A_{\mathrm{B}}}{A_{\mathrm{W}}}\right)^{1 / n}
$$

where $A_{\mathrm{B}}, A_{\mathrm{W}}$ and $n$ are respectively the creep coefficients for base metal, weld metal and creep exponent for power-law creep equation given as below

$$
\begin{gathered}
\dot{\varepsilon}_{\mathrm{B}}=A_{\mathrm{B}} \sigma^{n} \\
\dot{\varepsilon}_{\mathrm{W}}=A_{\mathrm{W}} \sigma^{n}
\end{gathered}
$$

in which $\dot{\varepsilon}_{\mathrm{B}}$ and $\dot{\varepsilon}_{\mathrm{W}}$ are the creep strain rates of base metal and weld metal, respectively.

For creeping weldments, a lot of discussions were focused on the creep stress distribution, e.g. Lee et al. [8] gave a study on the quantification of creeping stress of a welded branched pipe. Han et al. [9] also presented the creeping stress distribution of a welded branched pipe junction with a heat affected zone (HAZ). There were also some other related works on creeping weldments [10-12]. Besides the stress analysis of creeping components, the constraint effect for creep crack was also found to have a great influence on the evaluation of stress field [13]. Recently, some constraint parameters were proposed to characterize the constraint effect for creep crack, e.g. $Q$ parameter [13-15], $A_{2}^{*}$-parameter [16], $R$-parameter [17], $R^{*}$ parameter [18] and $A_{\mathrm{c}}$-parameter [19]. However, there are so far few discussions on quantifying the constraint effect of mismatch creep crack. Dai et al. [20] presented the $M^{*}$-parameter as a constraint parameter for characterization of material constraint effect of creep crack, where a mismatched modified boundary layer (MMBL) model was used.

In fact, the boundary layer model was used widely in elasticity or plasticity materials $[21,22]$. For creeping materials, Matvienko et al. [23] used the modified boundary layer (MBL) model to investigate the in-plane constraint parameter $A_{2}^{*}$ and out-of-plane constraint parameter $T_{z}$ under different $T$-stresses. Though some works like Refs. [20, 23] were presented, the influence of $T$-stress on the mismatched creep crack has not been investigated thoroughly. Especially, the influence of $T$-stress on the $Q$-parameter under creeping regime is still unknown yet. In this paper, the influence of $T$-stress on constraint effect of mismatch creep crack in MMBL model is studied.

\section{MISMATCHED MODIFIED BOUNDARY LAYER MODEL AND NUMERICAL PROCEDURE}

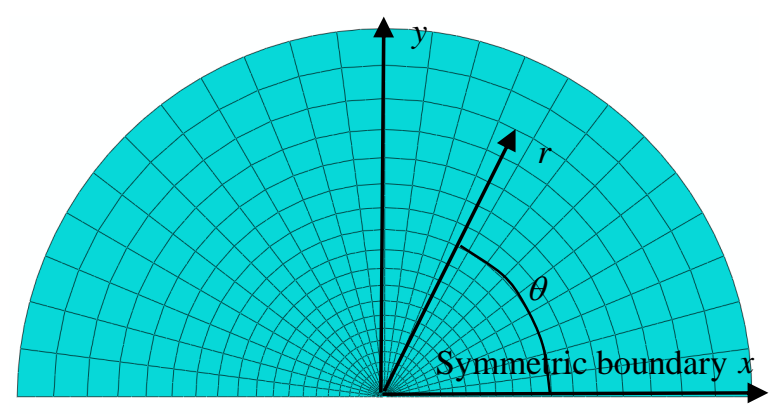

Fig. $1 \mathrm{FE}$ grid of MMBL model with creep crack

The MMBL model is carried out with a circular disc shown in Fig. 1, where the $x$ and $y$-axis coordinates are also presented. A half model is used here because of the symmetry of the MMBL model. The boundary conditions can be referred to Ref. [20], which are governed by analytical solutions for displacements of mode I crack with elastic field at the outer boundary. The displacements on the outer boundary of the MMBL model can be written as

$$
\begin{aligned}
u_{x}= & \frac{K_{\mathrm{I}}}{E} \sqrt{\frac{r}{2 \pi}}(1+v)(3-4 v-\cos \theta) \cos \frac{\theta}{2} \\
& +\frac{T}{E}\left(1-v^{2}\right) r \cos \theta \\
u_{y} & =\frac{K_{\mathrm{I}}}{E} \sqrt{\frac{r}{2 \pi}}(1+v)(3-4 v-\cos \theta) \sin \frac{\theta}{2} \\
& -\frac{T}{E} v(1+v) r \sin \theta
\end{aligned}
$$


where $K_{\mathrm{I}}$ is the stress intensity factor (SIF) of linear elasticity for mode I crack, $v$ is the Poisson's ratio and $r$ and $\theta$ are the polar coordinates also shown in Fig. 1. $T$ is the applied $T$ stress on the outer boundary of the MBL model. According to the MBL model, the small scale yielding can be obtained if the circular radius is large enough. Considering the similarity of HRR singularity [24] between power law elastoplastic field and power law elastic-creep field, the small scale creep can be obtained if the circular radius is large enough and the $T$-stress is not high, which is demonstrated by Dai et al. [25].

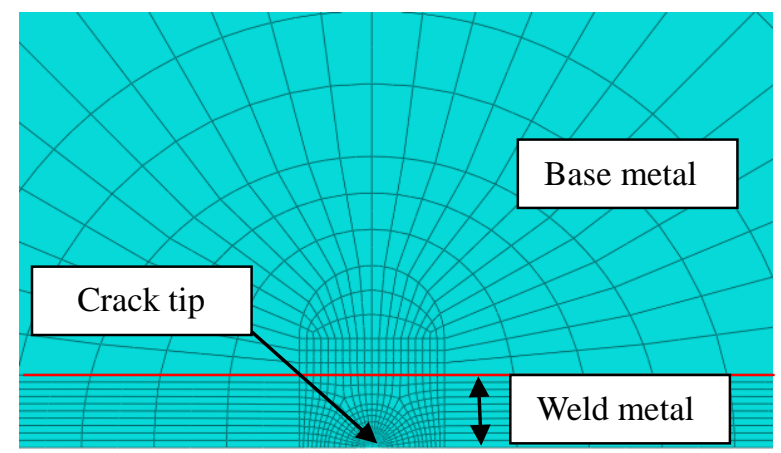

Fig. 2 FE mesh of crack tip for MMBL model

The finite element (FE) code ABAQUS is adopted here to perform the numerical analyses. The CPE8R is selected as the element type to simulate the crack tip. The detailed crack tip mesh can be seen in Fig. 2. The height of the weld metal is taken as $1.0 \mathrm{~mm}$, and a half-length of $0.5 \mathrm{~mm}$ is shown in Fig. 2. The total element number of the MMBL in this paper is 1844 . The material constants of P92 steel [26] for elastic power-law creep are adopted here, which can be seen in Table 1. For the analyzed cases, the creep exponents are kept as 5.23 and 7, respectively. The elastic modulus $E$, yielding stress $\sigma_{0}$ and Poisson's ratio are taken as $125000 \mathrm{MPa}, 180 \mathrm{MPa}$ and 0.3 , respectively.

Table 1 Creep material constants used in the calculation

\begin{tabular}{cccc}
\hline$A_{\mathrm{B}}\left(\mathrm{MPa}^{-n} \cdot h^{-1}\right)$ & $A_{\mathrm{W}}\left(\mathrm{MPa}^{-n} \cdot h^{-1}\right)$ & $M_{\mathrm{C}}$ & $n$ \\
\hline $2.64 \mathrm{E}-16$ & $2.64 \mathrm{E}-14$ & 0.41 & 5.23 \\
$2.64 \mathrm{E}-16$ & $2.64 \mathrm{E}-16$ & 1.00 & 5.23 \\
$2.64 \mathrm{E}-16$ & $2.64 \mathrm{E}-18$ & 2.41 & 5.23 \\
$3.20 \mathrm{E}-19$ & $1.60 \mathrm{E}-18$ & 0.79 & 7 \\
$3.20 \mathrm{E}-19$ & $3.20 \mathrm{E}-19$ & 1.00 & 7 \\
$3.20 \mathrm{E}-19$ & $6.40 \mathrm{E}-20$ & 1.26 & 7 \\
\hline
\end{tabular}

The applied far-field boundary conditions with mode I crack are used to predict the near crack tip stress field of MMBL model. To verify the accuracy of the applied boundary conditions, a comparison is made with the predicted elastic crack tip field in Table 2. It can be seen that the relative errors between the applied SIF and the predicted SIF are much lower than 10\%, which is always chosen as the upper error bound for the MBL model estimation. During the whole analysis, the applied SIF is kept as $100 \mathrm{MPa} \cdot \mathrm{mm}^{1 / 2}$.
Table $2 \mathrm{~A}$ comparison between the applied and predicted SIF

\begin{tabular}{ccc}
\hline $\begin{array}{c}\text { Applied SIF } \\
\left(\mathrm{MPa} \cdot \mathrm{mm}^{1 / 2}\right)\end{array}$ & $\begin{array}{c}\text { Predicted SIF } \\
\left(\mathrm{MPa} \cdot \mathrm{mm}^{1 / 2}\right)\end{array}$ & $\begin{array}{c}\text { Relative } \\
\text { Error }\end{array}$ \\
\hline 100 & 101.9 & $1.90 \%$ \\
150 & 152.8 & $1.86 \%$ \\
200 & 203.7 & $1.85 \%$ \\
\hline
\end{tabular}

\section{VARIATIONS OF T-STRESSES}

In general case, the $T$-stress of near field for creep crack can be presented as

$$
T=\sigma_{x x}-\sigma_{y y} \text { for } \theta=0^{\circ}
$$

where $\sigma_{x x}$ and $\sigma_{y y}$ are the stress of creep crack front in $x$ direction and $y$-direction, respectively. The variations of dimensionless $T$-stress with $r$ under the mismatch conditions are presented in Fig. 3. The $T$-stress in the legend represents the applied $T$-stress on the outer boundary. It can be found that the variations of $T$-stress for near field is dependent on mismatch factors, and dimensionless $T$-stress under the same mismatch factor presents the same variation tendency. Under the lower match condition, the dimensionless $T$-stress coincides well for different $T$-stresses of near crack tip where $r<1 \mathrm{~mm}$. If the $r$ exceeds the creep zone, the dimensionless $T$-stress of near crack tip here agrees well with the applied $T$-stress on the outer boundary. It implies that the stress field of MMBL on the mismatch condition has the same nature as the homogeneous condition, and it also can support that the MMBL in creep range is still valid here.

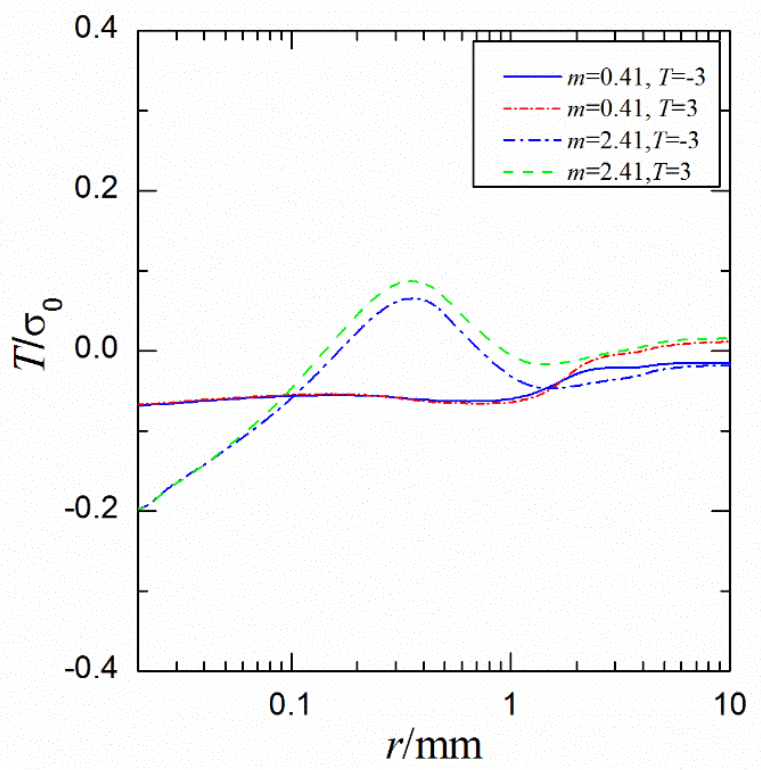

Fig. 3 Variations of dimensionless $T$-stresses under the undermismatch condition for different $T$-stresses 


\section{SELF-SIMILAR VERIFICATION OF MISMATCH CREEP CRACK STRESS FIELD}

The self-similar property for the mismatched creep crack tip has been verified by Dai et al. [20] with a normalized stress field. In this paper, we present the creep strain distributions of crack for different mismatch factors under $T=0$. Herein, the creep zone size is shown in Fig. 4 at creep time of 100000 hours with equivalent creep strain (CEEQ) of 1E-4. It can be seen that the creep zone size under the over-match condition with mismatch factor of 2.41 is much smaller compared with that under the even-match and the under-match conditions. In weld metal, the creep zone size under the under-match condition is much higher than that under the over-match condition. The above reason is that the material constraint under the over-match condition has the higher constraint value than that under the under-match and even-match conditions, and the higher constraint level restricts the creep zone in a small region. By the comparison of creep zone at different creep time, the distribution of CEEQ can explain clearly the self-similar property of the MMBL model.

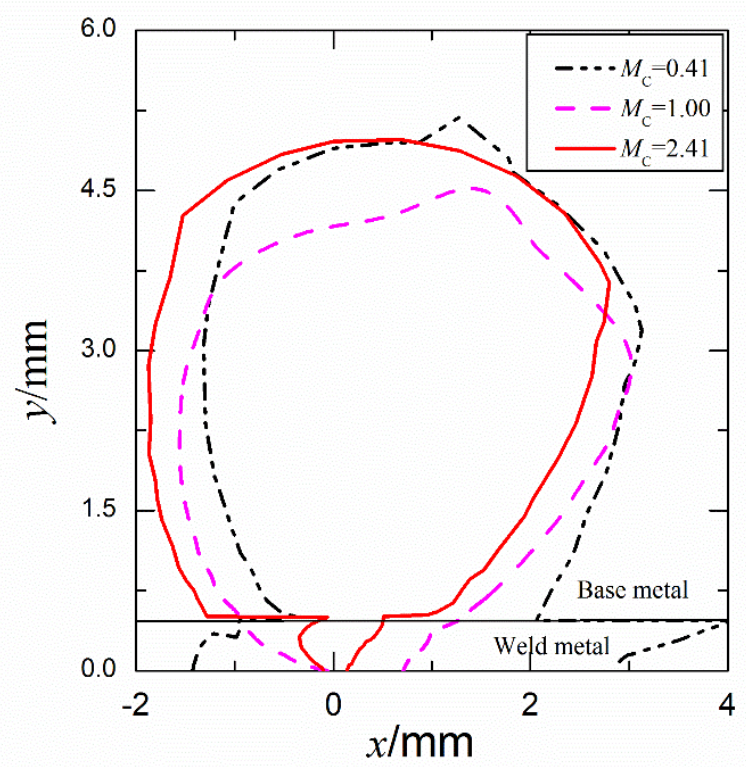

Fig. 4 CEEQ zone under different mismatch factors

\section{T-STRESS EFFECT ON CONSTRAINT OF MISMATCHED CREEP CRACK}

As a matter of fact, the $T$-stress term is obtained from the Williams' expansions [27]. For the MMBL model, the region near the crack tip is dominated by the creep zone compared with that of far field controlled by the elastic zone, which was demonstrated by Riedel and Rice [28]. Because of the analogy between the HRR field $[29,30]$ and the elastic power-law field, the stress field of creep zone for MBL model obeys the HRR singularity. According to the HRR field, the stress field of creep crack under the dominance of $C(t)$ in transient creep, or $C^{*}$ integral in extensive creep, can be written as

$$
\begin{gathered}
\sigma_{i j}(r, \theta)=\sigma_{0}\left(\frac{C(t)}{\sigma_{0} \dot{\varepsilon}_{0} I_{n} r}\right)^{1 /(n+1)} \tilde{\sigma}_{i j}(n, \theta) \\
\sigma_{i j}(r, \theta)=\sigma_{0}\left(\frac{C^{*}}{\sigma_{0} \dot{\varepsilon}_{0} I_{n} r}\right)^{1 /(n+1)} \tilde{\sigma}_{i j}(\theta)
\end{gathered}
$$

where $\sigma_{0}, \dot{\varepsilon}_{0}, I_{n}, \quad r$ and $\tilde{\sigma}_{i j}(\theta)$ are yielding stress, reference creep strain rate, integral constant, distance from crack tip and angular function, respectively.

If the constraint effect is taken into consideration, the stress field for elastic power-law creep, can be written as [13]

$$
\sigma_{i j}(r, \theta)=\sigma_{0}\left(\frac{C^{*}}{\sigma_{0} \dot{\varepsilon}_{0} I_{n} r}\right)^{1 /(n+1)} \tilde{\sigma}_{i j}(n, \theta)+Q \sigma_{0} \delta_{i j}
$$

where $Q$ is the creeping constraint parameter similar to elastoplastic constraint parameter given by Shih et al. [31], and $\delta_{i j}$ is the Kronecker's delta. Generally, the constraint parameter $Q$ can be calculated as follows [31]:

$$
\begin{gathered}
Q=\frac{\sigma_{22}-\sigma_{22}^{\mathrm{HRR}}}{\sigma_{0}} \\
Q=\frac{\sigma_{22}-\sigma_{22}^{\mathrm{SSY}, T=0}}{\sigma_{0}}
\end{gathered}
$$

where $\sigma_{22}^{\mathrm{HRR}}$ and $\sigma_{22}^{\mathrm{SSY}, T=0}$ are the open stress (or tangential stress) of HRR field under small scale yielding with $T=0$. As for the elastoplastic material, the relationship between the $Q$ parameter and $T$-stress can be described by [31]

$$
Q=a_{1}\left(\frac{T}{\sigma_{0}}\right)^{1}+a_{2}\left(\frac{T}{\sigma_{0}}\right)^{2}+a_{3}\left(\frac{T}{\sigma_{0}}\right)^{2}
$$

where $a_{1}, a_{2}$ and $a_{3}$ are coefficients depending on loading level and material properties.

As the stress field of creep crack can be affected by the $C(t)$ integral, the $C(t)$-integrals under different mismatch factors are firstly discussed in Fig. 5. It implies that the $C(t)$-integral on the under-match condition is higher than the $C(t)$-integral on the even-match condition for short creep time. If the creep time is long enough, the $C(t)$-integral approaches to be nearly the same. 


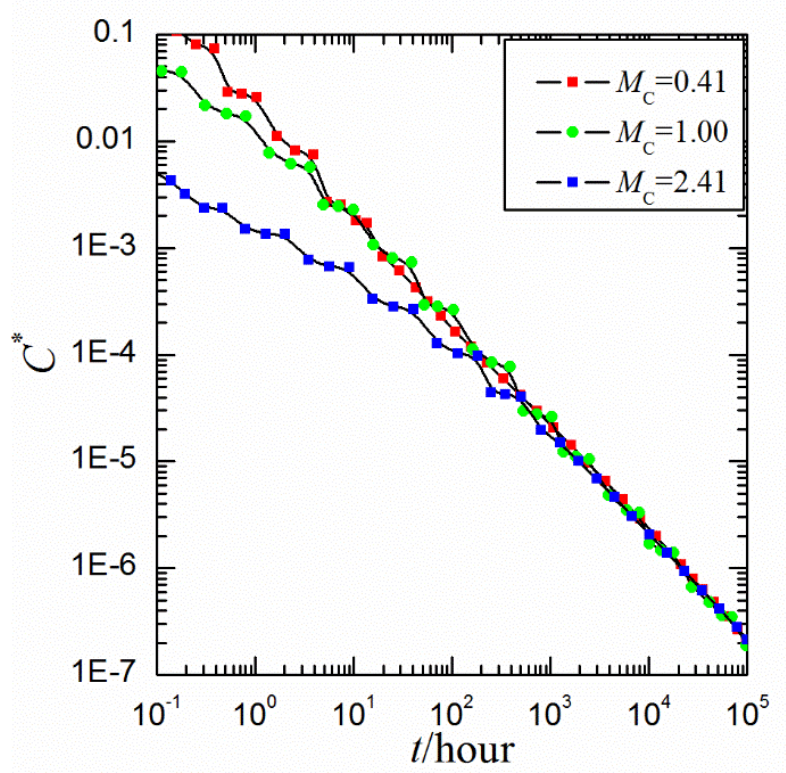

Fig. 5 Variations of $C^{*}$-integrals with creep time under different mismatch factors

With Eqs. (7)-(8), the open stresses of analytical HRR field at 100000 hours are shown in Fig. 5. It can be seen that the open stress $\sigma_{22}$ under the even-match condition with $T=0$ coincides with analytical HRR field very well. However, the open stress on the under-match condition with mismatch factor of 0.41 is much lower than that under the even-match condition. The open stress on the over-match condition is larger than that under the evenmatch and under-match conditions. As the high constraint level on the over-match condition has a much smaller creep zone, the open stress near the crack tip on the over-match condition deviates from the HRR field greatly.

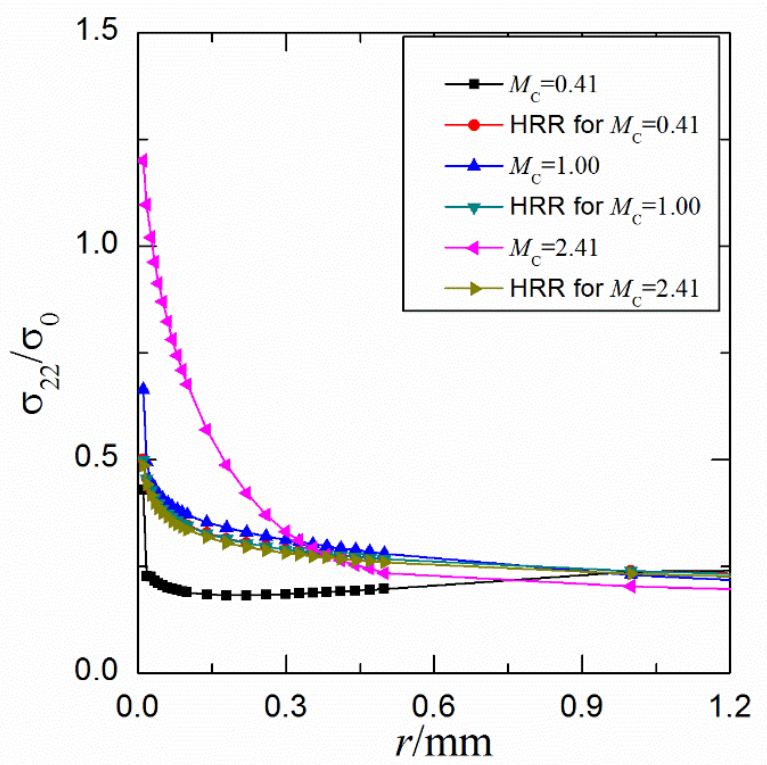

Fig. 6 Comparison of open stresses at long creep time for different mismatch factors with $T=0$
As the $C(t)$-integral under different mismatch factors at long creep time does not differ much from each other, the open stresses of analytical field presented in Fig. 6 agree well with each other. It should be pointed out that the differences between the open stresses of the analytical HRR field are significant if the creep time is very short.

To have a better understanding of the $T$-stress in the stress field of creeping crack tip, the open stresses of MMBL model under different mismatch factors with various $T$-stresses are presented in Figs. 7-9. It can be seen that the open stress of creep crack tip coincides with the HRR field perfectly only when $T=0$ under even-match condition. It implies that the stress field with $T=0$ can be used as the reference stress field to characterize the constraint effect of creep crack as Eq. (12). Under the evenmatch condition, the open stress of creep crack tip for negative $T$-stress is lower than that of the HRR field, and also lower than that of creep crack tip for positive $T$-stress, as shown in Fig. 7.

Under the under-match condition presented in Fig. 8, the open stresses of creep crack tip are lower than those of the HRR field for both negative $T$-stress and positive $T$-stress. The difference of open stresses between positive $T$-stress and negative $T$-stress is slight. Under the over-match condition shown in Fig. 9, the conclusion is quite similar to that under the under-match condition, however, the difference is that the open stress in creep zone on the over-match condition is much higher than that of the HRR field.

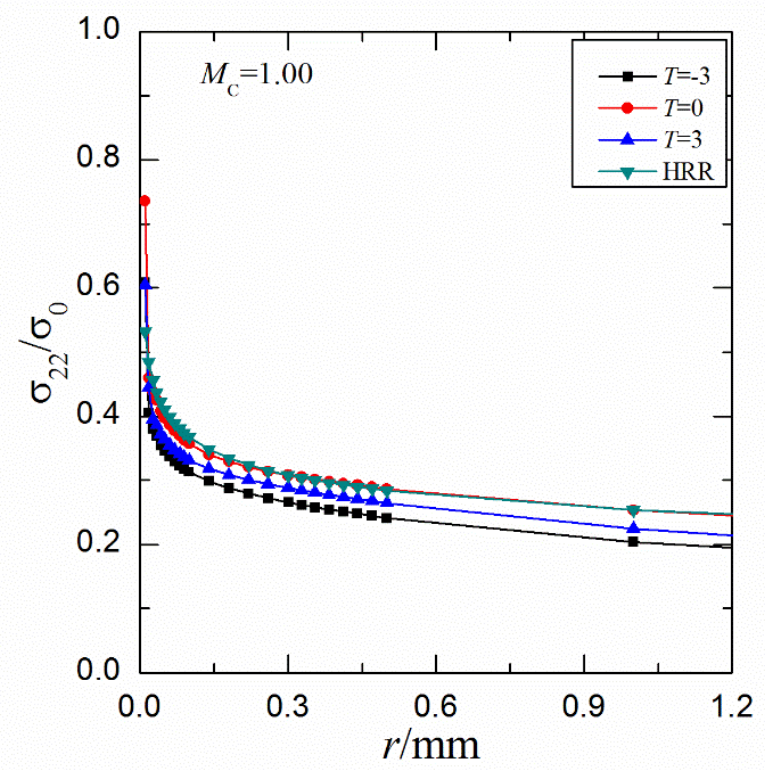

Fig. 7 Comparison of open stresses at long creep time on the even-match condition for different $T$-stresses 


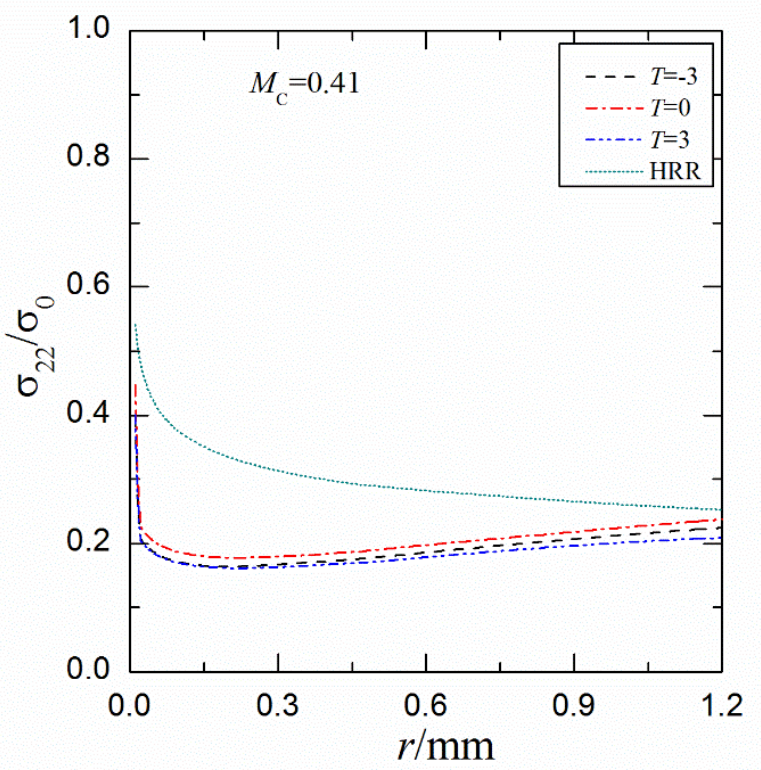

Fig. 8 Comparison of open stresses at long creep time on the under-match condition for different $T$-stresses

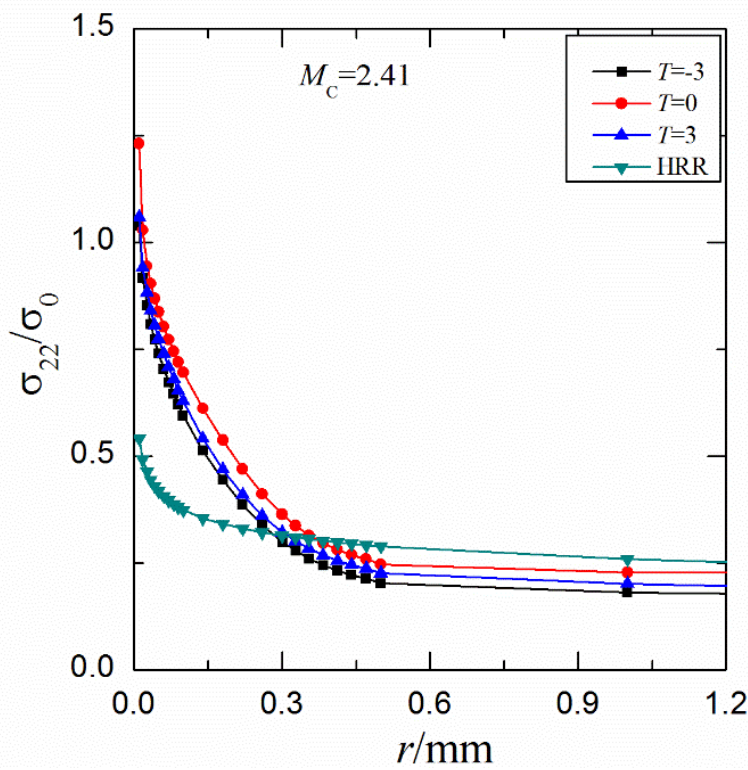

Fig. 9 Comparison of open stresses at long creep time on the over-match condition for different $T$-stresses

Based on the analysis of open stress, Eq. (11) is first used to characterize the constraint effect of mismatched creep crack in the MMBL model (see Fig. 10). It can be seen that positive values of constraint parameter $Q$ of creep crack are obtained on the over-match condition, while negative values are gotten on the under-match condition.

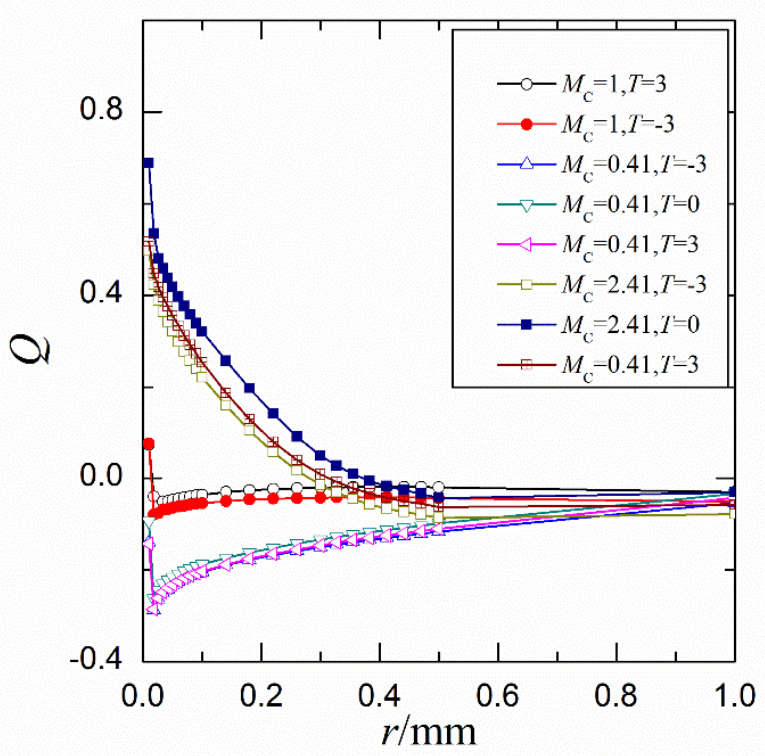

Fig. 10 Variations of $Q$ for different mismatch factors and $T$ stresses

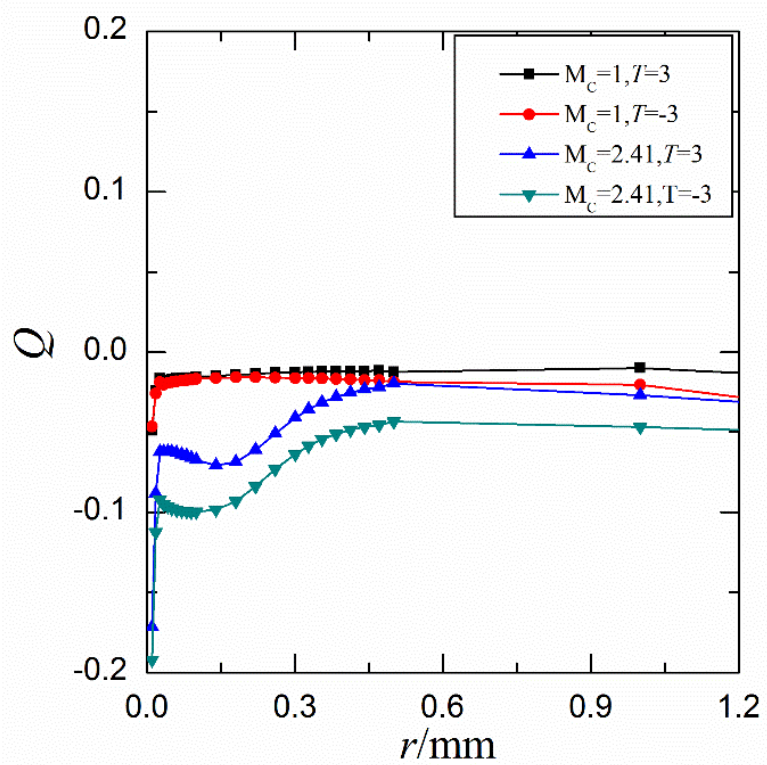

Fig. 11 Variations of $Q$ for different mismatch factors and $T$ stresses

Instead of Eqs. (11)-(12), a formula to characterize the constraint effect of mismatched creep crack is proposed as

$$
Q=\frac{\sigma_{22}(M)-\sigma_{22}^{\mathrm{SSC}, T=0}(M)}{\sigma_{0}}
$$

where $\sigma_{22}(M)$ means the open stress of mismatched creep crack with $T \neq 0$ and $\sigma_{22}^{\mathrm{SSC}, T=0}(M)$ represents the open stress of mismatched creep crack with $T=0$ under small scale creep. With the definition of Eq. (14), the constraint effect caused by loading 
condition can be obtained (see Fig 11). According to Dai et al. [20], Eq. (14) is the constraint effect caused by generalized geometry (loading mode and geometric size), and the constraint effect of material is characterized by the material constraint parameter $M^{*}$. It can be seen that for a mismatched creep crack in the MMBL model, the material mismatch constraint effect plays a more significant role than the geometric constraint effect. The role of $T$-stress can affect the geometric constraint only.

Creep is time-dependent, and the behavior of creep relaxation is the most important difference between creep and plasticity. Figs. 12-13 present the variations of open stress with time on the over-match condition and the under-match condition. The open stresses given here are obtained at the same distance from crack tip with $r=0.1 \mathrm{~mm}$ in the creep zone. It can be seen that the variations of open stress on the over-match and the under-match conditions are rather different. Under the overmatch condition, the influence of $T$-stress on open stress of creep crack tip is not significant, however, there are blunting near the crack tip. Under the under-match condition, the influence of $T$ stress on open stress is slight. The above reason is that the creep relaxation under the over-match condition is significant as the creep crack tip has a higher stress level. Under the under-match condition, the influence of $T$-stress on the stress level of creep crack tip is not significant like that under the over-match condition.

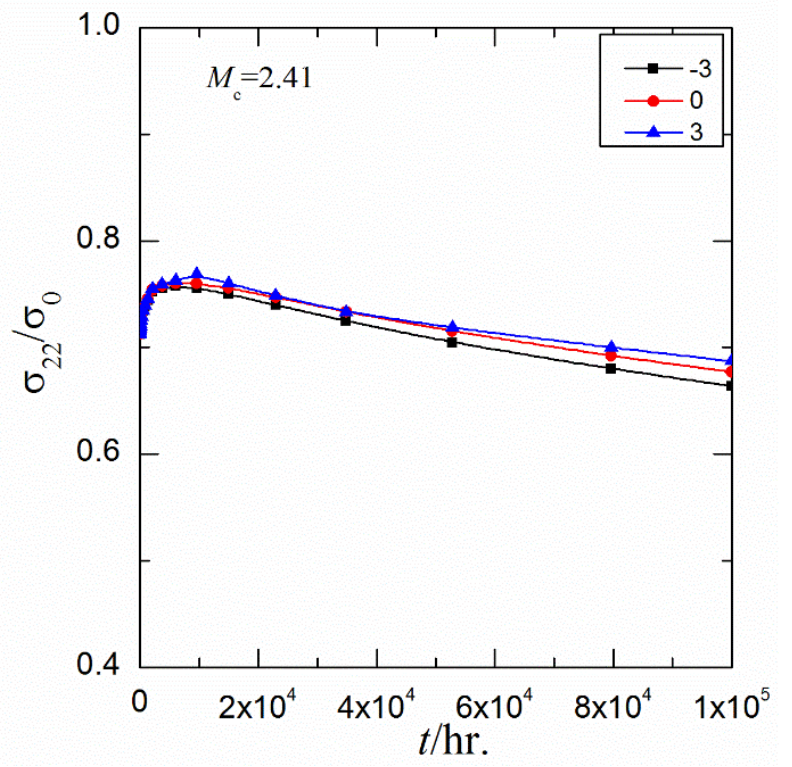

Fig. 12 Variations of dimensionless open stress under the overmismatch condition for different $T$-stresses

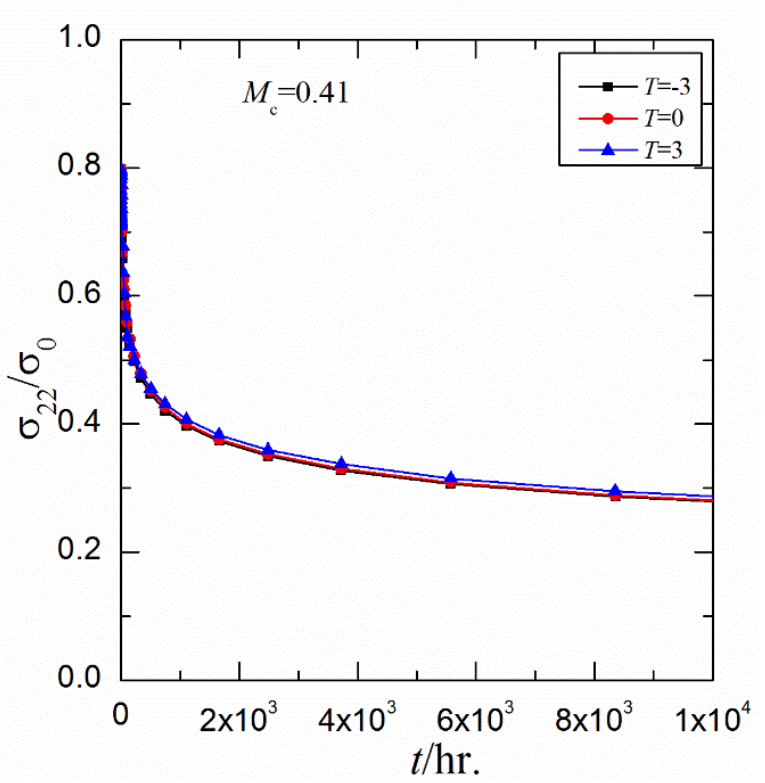

Fig. 13 Variations of dimensionless open stress under the undermismatch condition for different $T$-stresses

\section{INFLUENCE OF CREEP EXPONENT}

As the creep exponent is also significant on the influence of the creep behavior, the investigation on the influence of creep exponent. The creep constant for $n=7$ is used here where the specific constants can be seen from Table 1. Herein, three different mismatch factors, i.e. $m=0.83,1.00$ and 1.39 , have the different creep coefficients compared with $n=5.23$. The variations of dimensionless open stress under different mismatch factors for different creep exponent are presented in Fig.14. The interesting thing is that the open stresses here have the same tendencies for $m=1.0$ though the creep coefficients and creep exponents differ much. The open stress level increases with the enhancement of mismatch factors, and it relies on mismatch factors only from this evidence. 


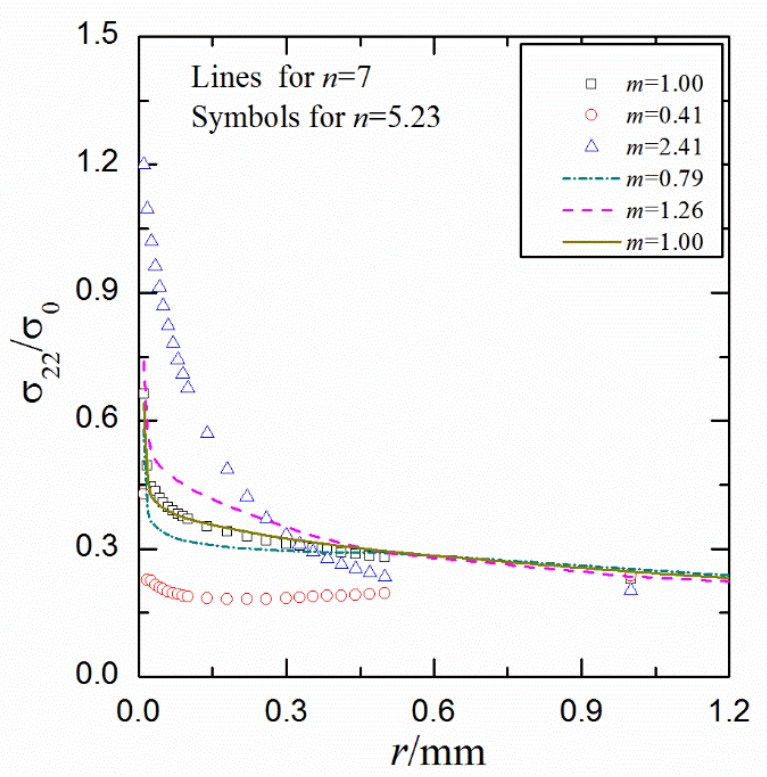

Fig. 14 Variations of dimensionless open stress under the under different $T$-stresses for different creep exponents

\section{CONCLUDING REMARKS}

A MMBL model is adopted to investigate the influences of $T$-stress on constraint effect of creep crack in this paper. The stress field of creep crack tip in the MMBL model under different mismatch factors are presented here. The characterization of constraint effect for creep crack under the mismatch condition is given. According to the above study, the conclusions can be obtained as follows:

1) The variations of $T$-stress in creep regime is presented. Results show that the $T$-stress under the same mismatch factor has the tendency. The stress field of creep crack in MMBL model is combined by HRR type field in creep range and elastic field of far field. The MMBL is valid to be used under the creep regime.

2) The creep zone size of crack is rather different under different mismatch factors. It implies the creep zone size on the mismatch condition is affected remarkably by the mismatch factor. It demonstrates that creep crack on under-match condition has the larger creep zone than that on the over-match condition. That shows the material mismatch with higher constraint effect can restrict the creep zone, which is very similar to elastoplastic materials.

3) It can be found that the influence of $T$-stress on constraint effect of creep crack within the MMBL model is quite different from elastoplastic materials. However, the negative or positive $T$-stress can still influence the geometric constraint effect of creep crack. As usual, the negative $T$-stress can lead to lower open stress and has lower constraint effect. Compared with the material mismatch constraint, the influence of
$T$-stress on geometric constraint effect seems to be not significant as expected. The applications of this conclusion to the specimens or structures need a further study.

4) Under the over-match condition, there is blunting effect though the $T$-stress is under small range. Under the under-match condition, the influence of $T$-stress on open stress is not remarkable if the $T$-stress is not large enough. Both positive and negative $T$-stresses can accelerate the creep relaxation of creep crack tip field. Under the mismatch condition, the $C(t)$-integrals are really different during short creep time for different mismatch factors, however, they behave similarly if the creep time is long enough.

5) The open stresses are the same though the creep exponents and creep coefficients differ much. It implies that the level of open stress is dependent on the mismatch factor for the same loading.

\section{ACKNOWLDGMENT}

This work was supported by the National Science Foundation for Distinguished Young Scholars of China (Grant No. 11325211) and the Project of International Cooperation and Exchange NSFC (Grant No. 11511130057).

\section{REFERENCES}

[1] Haddi, A., and Weichert, D., 1997, "Elastic-plastic J-integral in inhomogeneous materials," Computational Materials Science, 8(3), pp. 251-260.

[2] Kim, Y.-J., and Schwalbe, K.-H., 2001, "Mismatch effect on plastic yield loads in idealised weldments: II. Heat affected zone cracks," Engineering Fracture Mechanics, 68(2), pp. 183-199.

[3] Kim, Y.-J., and Schwalbe, K.-H., 2001, "Mismatch effect on plastic yield loads in idealised weldments: I. Weld centre cracks," Engineering Fracture Mechanics, 68(2), pp. 163-182.

[4] Song, T.-K., Kim, Y.-J., Kim, J.-S., and Jin, T.-E., 2008, "Mismatch limit loads and approximate $\mathbf{J}$ estimates for tensile plates with constant-depth surface cracks in the center of welds," International Journal of Fracture, 148(4), pp. 343-360.

[5] Zhang, Z. L., Hauge, M., and Thaulow, C., 1996, "Twoparameter characterization of the near-tip stress fields for a bimaterial elastic-plastic interface crack," International Journal of Fracture, 79(1), pp. 65-83.

[6] Thaulow, C., Zhang, Z., and Hauge, M., 1997, "Effects of crack size and weld metal mismatch on the has cleavage toughness of wide plates," Engineering Fracrure Mechanics, 57(6), pp. 653-664.

[7] Thaulow, C., Zhang, Z., Hauge, M., Burget, W., and Memhard, D., 1999, "Constraint effects on crack tip stress fields for cracks located at the fusion line of weldments," Computational Materials Science, 15(3), pp. 275-284.

[8] Lee, K. H., Kim, Y. J., Yoon, K. B., Nikbin, K., and Dean, D., 2010, "Quantification of stress redistribution due to mismatch in creep properties in welded branch pipes," Fatigue \& Fracture of Engineering Materials \& Structures, 33(4), pp. 238-251. 
[9] Han, J. J., Kim, Y. J., Jerng, D., Nikbin, K., and Dean, D., 2015, "Quantification of creep stresses within HAZ in welded branch junctions," Fatigue \& Fracture of Engineering Materials \& Structures, 38(1), pp. 113-124.

[10] Dogan, B., and Petrovski, B., 2001, "Creep crack growth of high temperature weldments," International Journal of Pressure Vessels and Piping, 78(11-12), pp. 795-805.

[11] Segle, P., Andersson, P., and Samuelson, L. Å., 2000, "Numerical investigation of creep crack growth in cross-weld CT specimens. Part I: influence of mismatch in creep deformation properties and notch tip location," Fatigue \& Fracture of Engineering Materials \& Structures, 23(6), pp. 521531.

[12] Yoon, K. B., and Kim, K. Y., 1999, "High temperature fracture parameter for a weld interface crack," Theoretical and Applied Fracture Mechanics, 32(1), pp. 27-35.

[13] Budden, P., and Ainsworth, R., 1997, "The effect of constraint on creep fracture assessments," International Journal of Fracture, 87(2), pp. 139-149.

[14] Nikbin, K., 2004, "Justification for meso-scale modelling in quantifying constraint during creep crack growth," Materials Science and Engineering: A, 365(1-2), pp. 107-113.

[15] Yatomi, M., and Tabuchi, M., 2010, "Issues relating to numerical modelling of creep crack growth," Engineering Fracture Mechanics, 77(15), pp. 3043-3052.

[16] Chao, Y., Zhu, X., and Zhang, L., 2001, "Higher-order asymptotic crack-tip fields in a power-law creeping material," International Journal of Solids and Structures, 38(21), pp. 38533875 .

[17] Wang, G. Z., Liu, X. L., Xuan, F. Z., and Tu, S. T., 2010, "Effect of constraint induced by crack depth on creep crack-tip stress field in CT specimens," International Journal of Solids and Structures, 47(1), pp. 51-57.

[18] Tan, J., Tu, S., Wang, G., and Xuan, F., 2013, "Effect and mechanism of out-of-plane constraint on creep crack growth behavior of a $\mathrm{Cr}-\mathrm{Mo}-\mathrm{V}$ steel," Engineering Fracture Mechanics, 99, pp. 324-334.

[19] Ma, H. S., Wang, G. Z., Xuan, F. Z., and Tu, S. T., 2015, "Unified characterization of in-plane and out-of-plane creep constraint based on crack-tip equivalent creep strain," Engineering Fracture Mechanics, 142, pp. 1-20.

[20] Dai, Y., Liu, D., and Liu, Y., 2016, "Mismatch constraint effect of creep crack with modified boundary layer model,"
ASME Journal of Applied Mechanics, 83(3), pp. 031008031016

[21] Larsson, S.-G., and Carlsson, A. J., 1973, "Influence of nonsingular stress terms and specimen geometry on small-scale yielding at crack tips in elastic-plastic materials," Journal of the Mechanics and Physics of Solids, 21(4), pp. 263-277.

[22] Jayadevan, K. R., Narasimhan, R., Ramamurthy, T. S., and Dattaguru, B., 2002, "Effect of T-stress and loading rate on crack initiation in rate sensitive plastic materials," International Journal of Solids and Structures, 39(7), pp. 1757-1775.

[23] Matvienko, Y. G., Shlyannikov, V., and Boychenko, N., 2013, "In - plane and out - of - plane constraint parameters along a three - dimensional crack - front stress field under creep loading," Fatigue \& Fracture of Engineering Materials \& Structures, 36(1), pp. 14-24.

[24] Hoff, N., 1954, "Approximate analysis of structures in the presence of moderately large creep deformations," Quarterly of Applied Mathematics, 12(1), pp. 49.

[25] Dai, Y., Liu, D., Liu, Y., and Chen, H., 2016, "Influence of T-Stress on Creep Crack Tip Field. Part A: Mode I Crack," International Journal of Solids and Structures, to be submitted.

[26] Zhao, L., Xu, L., Han, Y., and Jing, H., 2015, "Twoparameter characterization of constraint effect induced by specimen size on creep crack growth," Engineering Fracture Mechanics, 143, pp. 121-137.

[27] Williams, M., 1957, "On the stress distribution at the base of a stationary crack." ASME Journal of Applied Mechanics, 24, pp. 111-114.

[28] Riedel, and Rice, 1980, "Tensile cracks in creeping solids," West Conshohocken, PA: ASTM STP, 700, pp. 112-130.

[29] Hutchinson, J., 1968, "Singular behaviour at the end of a tensile crack in a hardening material," Journal of the Mechanics and Physics of Solids, 16(1), pp. 13-31.

[30] Rice, J., and Rosengren, G., 1968, "Plane strain deformation near a crack tip in a power-law hardening material," Journal of the Mechanics and Physics of Solids, 16(1), pp. 1-12.

[31] Shih, C. F., O'Dowd, N., and Kirk, M., 1991, "A framework for quantifying crack tip constraint," Constraint Effects in Fracture, pp. 2-20. 